\title{
R\&D Studies of the ATLAS LAr Calorimeter Readout Electronics for super-LHC
}

\author{
H. Chen ${ }^{\mathrm{a}, *}$, on behalf of the ATLAS Liquid Argon Calorimeter Group \\ ${ }^{a}$ Brookhaven National Laboratory, Upton, NY 11973-5000, U.S.A.
}

\begin{abstract}
The ATLAS Liquid Argon (LAr) calorimeters are high precision, high sensitivity and high granularity detectors. A total of 182,468 signals are digitized and processed real-time on detector, to provide energy and time deposited in each detector element at every occurrence of the L1-trigger. The current readout electronics will need to be upgraded to sustain the higher radiation levels expected with the increase of a factor 10 in luminosity at the LHC in the years $\sim 2017$. A completely innovative readout scheme is being developed. The front-end readout will send out data continuously at each bunch crossing through high speed radiation resistant optical links, the data will be processed real-time with the possibility of implementing trigger algorithms.

This article is an overview of the R\&D activities and architectural studies the ATLAS LAr Calorimeter Group is developing: front-end analog and mixed-signal ASIC design, radiation resistance optical-links in SOS, high-speed back-end processing units based on FPGA architectures and power supply distribution schemes.
\end{abstract}

Key words:

Liquid argon calorimeter, Readout electronics, Readout driver, Radiation hardness

\section{Introduction}

The ATLAS experiment [1] is one of the two general purpose detectors designed to study the proton-proton collisions produced at the Large Hardron Collider (LHC) with a center-of-mass energy of $14 \mathrm{TeV}$ and to explore the full physics potential of the LHC at CERN.

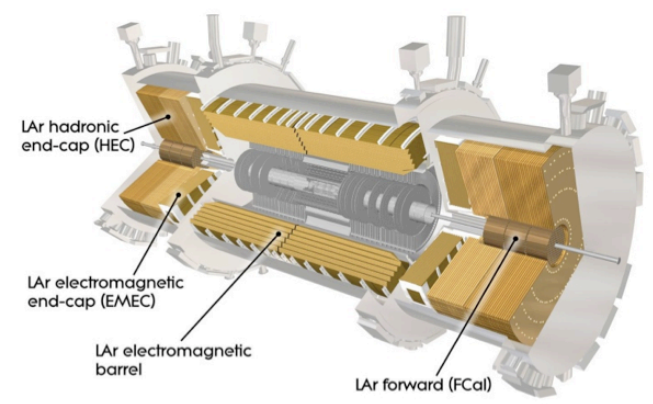

Figure 1: Schematic drawing of the ATLAS LAr calorimeters

Liquid Argon (LAr) calorimeters [2] in ATLAS are high precision, high sensitivity and high granularity detectors designed to provide precision measurements of electrons, photons, jets and missing transverse energy. The LAr calorimeters consist of four sub-detectors and are contained within three cryostats as shown in Figure 1. The central cryostat houses the electromagnetic barrel calorimeter

\footnotetext{
*Corresponding author. Tel.: +1-631-344-3468; fax: +1-631-3445078 .

Email addresses: chc@bnl.gov (H. Chen)
}

(EMB) which covers the pseudo-rapidity range $0<|\eta|<$ 1.475 , while each end-cap cryostat contains an end-cap electromagnetic calorimeter (EMEC) which covers $1.375<$ $|\eta|<3.2$, two hadronic end-cap wheels (HEC) covering $1.5<|\eta|<3.2$ and a three wheels forward calorimeter (FCAL) which covers the region very close to the beam axis $(3.1<|\eta|<4.9)$ to achieve large acceptance.

\section{LAr Calorimeter Readout Electronics}

The readout electronics architecture of LAr calorimeters is shown in Figure 2. The ionization current signal is preamplified, shaped, buffered and digitized by the frontend boards (FEB) [3] installed on the detector. The FEBs send the digitized pulse via optical links to the Readout Drivers (ROD) which are installed in a radiation free area (USA15) next to the detector cavern (UX15). In total 182,468 calorimeter cells are to be read out. Each ROD receives the data from up to 8 FEBs and calculates the energy deposited, the time of the deposition and a quality factor for cells with high energy deposit, using an optimal filtering algorithm [4]. RODs send data to the Readout Buffers (ROBs) hosted on PCs (ROS) through S-links. Analog trigger sum signals are formed on front-end electronics boards and sent to the receiver system through copper cables before interface to the Level-1 calorimeter trigger system.

The LAr calorimeter readout electronics can be divided into two sub-systems, front-end electronics [5] and back-end electronics [6] systems. Front-end electronics system is installed on detector and includes 58 Front End 


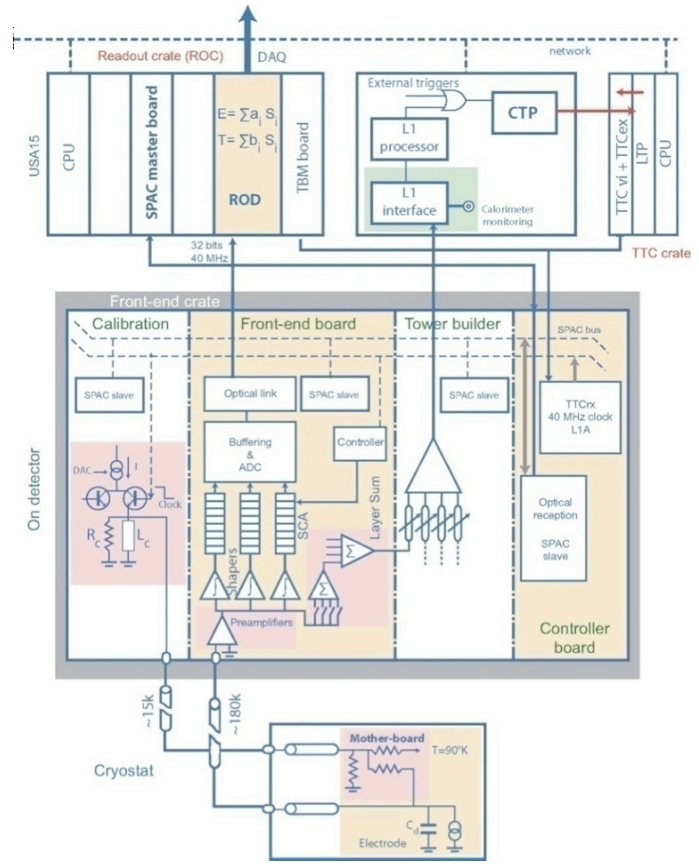

Figure 2: LAr calorimeters readout electronics architecture

Crates (FEC) which house 1,524 FEBs and other electronics boards. Each FEC has one radiation hardened Low Voltage Power Supply (LVPS) to provide power to electronics boards. There are 1,600 fiber optical links between front-end and back-end electronics systems. Backend electronics system consists of 16 back end crates and 68 ROS PCs, and there are total 192 RODs plugged in back end crates and $\sim 800$ fiber optical links between RODs and ROS.

\section{Upgrade Motivations}

LHC is expected to be upgraded in two phases. Phase 1 is planned in 2013 and phase 2 is planned in 2017 which is also known as super-LHC. Super-LHC will have 10 times luminosity and the current LAr front-end electronics need to be upgraded to sustain the higher radiation levels [7]. The present front-end architecture is very complex. A FEB has 11 ASICs based on different technologies, some of which (e.g. DMILL) are already obsolete. The FEB is designed and produced for 10 years operation with limited number $(\sim 6 \%)$ of spares, it has also intrinsic limitations on Level-1 trigger acceptance. Therefore component level upgrade is not possible, and a new front-end architecture has been proposed as shown in Figure 3.

The new front-end readout will send out data continuously at each bunch crossing through high speed radiation resistant optical links. The data (100Gbps each board) will be processed real-time with the possibility of implementing trigger algorithms for clusters and electron/photon identification at a higher granularity than what is currently implemented. The new architecture simplifies the system

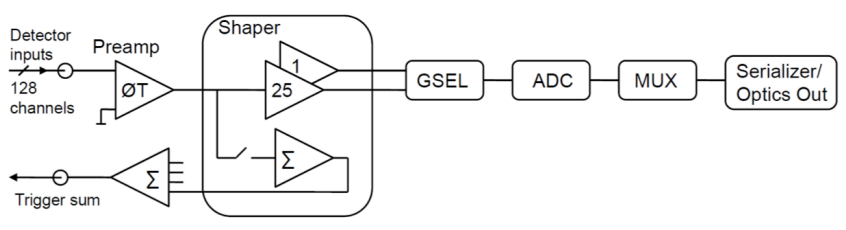

Figure 3: Proposed front-end readout architecture of LAr calorimeter

design while keeping many options open, such as pipeline design, shaping and gain settings etc. It requires many $R \& D$ efforts and the following section describes these $R \& D$ activities for LAr calorimeter readout electronics upgrade.

\section{R\&D Activities of Readout Electronics Upgrade}

\subsection{Front-end ASIC Design}

Front-end electronics design for super-LHC becomes more critical with requirements of both high performance and higher radiation level. Two R\&D efforts are going on which are focused on the analog front-end and mixedsignal front-end ASIC design respectively.

\subsubsection{Analog Front-end RED}

SiGe (silicon germanium) BiCMOS technology has drawn much attention in fast detector readout and applications where radiation is a concern. Both Inner Detector and LAr calorimeter communities are investigating SiGe for the ATLAS upgrade. Many characterization tests have been performed with foundry provided transistor samples in different radiation environments (gamma, neutron and proton irradiation). A test structure chiplet based on IBM 8WL $0.13 \mu \mathrm{m}$ design kit parts has been designed and submitted. It will be used to test radiation performance of different transistor configurations. This chiplet also has IBM 8WL CMOS transistor array on chip. A direct comparison with IBM $8 \mathrm{SF}$ CMOS only process can be done.

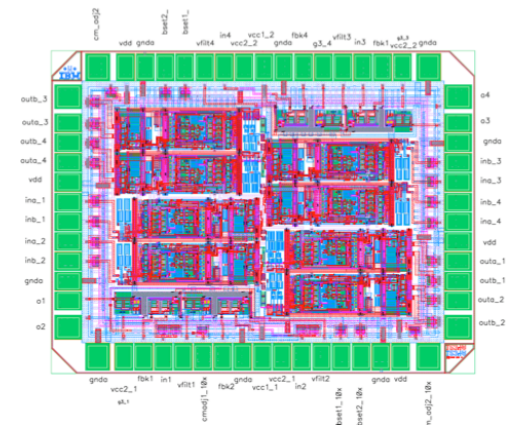

Figure 4: Layout of the LAr analog front-end ASIC design

An analog front-end chip which includes preamplifiers and shapers has been designed and submitted to MOSIS in Nov 2008. The preamplifier design is based on low noise line-terminating circuit topology presently used in 
LAr calorimeter. It has high dynamic range and very low noise $(\sim 0.25 n \mathrm{~V} / \sqrt{\mathrm{Hz}})$. Shaper design is fully differential and based on $C R-(R C)^{2}$ topology with two gain settings $(\times 1$ and $\times 10)$, and it has robust performance in low signal environment and excellent pickup rejection on and off chip. The layout of $2 \mathrm{~mm} \times 2.2 \mathrm{~mm}$ chiplet is shown in Figure 4.

\subsubsection{Mixed-signal Front-end: $A D C$}

ADC is the most technologically challenging component in the new architecture due to the requirements of high dynamic range, radiation tolerance and SEE (single event effects) immunity. We are planning to test some commercial ADCs which usually have bit error correction stored in radiation soft RAM, while a custom ADC is being developed. IBM 8RF CMOS technology has been chosen as a possible candidate technology for ADC design, which has shown to be radiation hard and has lower cost compared to SiGe. Many critical techniques, such as $\mathrm{S} / \mathrm{H}$ capacitor, amplifier and digital correction etc., have been studied for the pipeline ADC design, and a MOSIS test structure proposal is planned to be submitted by Jun 2009 .

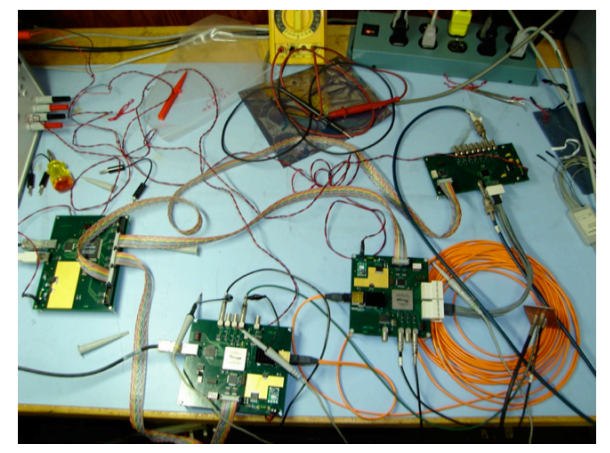

Figure 5: Test setup of initial digital test design

Digital logic design of new architecture has been tested in the lab (see Figure 5). This test utilizes two independent clocks, one of which is to emulate ATLAS TTC like clock for ADC and ADC multiplexer, and the other is crystal derived clock for high speed components to provide better jitter control. Some techniques that could be used to improve radiation hardness are implemented in this setup, and the full DAQ chain has been tested and debugged successfully.

\subsection{Radiation Resistance Optical Link}

The new readout architecture requires high bandwidth radiation hard optical link to transmit data out of the detector. $0.25 \mu \mathrm{m}$ UltraCMOS Silicon on Sapphire (SOS) technology has been chosen to design an optical link transceiv due to its inherent resistance to radiation. The characterization test was performed on a dedicated test chip, and TID (total ionization dose) and SEE test results show that the SOS process has very good radiation resistance.

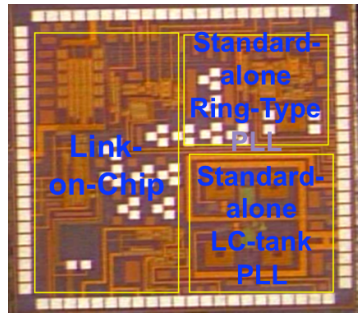

Figure 6: Photograph of the first prototype of Link on Chip

The first prototype of Link on Chip (LOC1) running at $2.5 \mathrm{Gbps}$ was available in Jun 2007. Figure 6 is the photograph of LOC1. Characterization test and SEE test were conducted. Large jitter is observed and understood during the lab test. The improved design of critical components (serializer and PLL) will be applied to the second prototype (LOC2) which will be able to interface to CERN Versatile Link. The LOC2 design is progressing steadily and submission is planned in Jun 2009.

\subsection{Back-end High-speed Processing Unit}

With the new front-end architecture, ROD will have to be upgraded to process huge amount of data from the detector. ROD upgrade R\&D is to prototype a ROD that can receive and process continuously digitized detector signals, at the same time we also investigate and evaluate several critical technologies which could be used in the design of next generation ROD. ROD R\&D interests include high speed commercial optical link and FPGA SERDES, low latency lossless data compression/decompression algorithm, FPGA based digital signal processing, performing Level-1 trigger sum digitally and exploring a different system level architecture (AdvancedTCA).

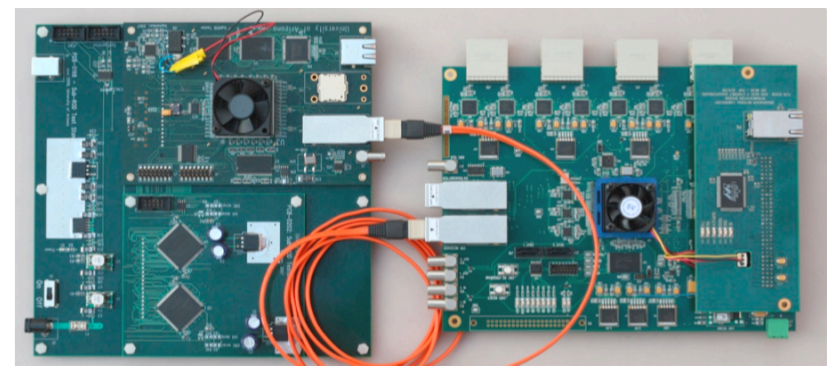

Figure 7: Sub-ROD and Sub-ROD Injector

The first version of Sub-ROD and Sub-ROD Injector shown in Figure 7 were developed and the integration test was performed in Sept 2008. They use commercial parallel optical transceiver running at 40Gbps with MPO/MTP low profile connector, SERDES of Xilinx Virtex-5 FPGA on Sub-ROD, and Altera Stratix II GX FPGA on SubROD Injector are tested successfully. A DSP algorithm is implemented in both Xilinx and Altera FPGAs. 
We are investigating the possibility to implement trigger algorithms for clusters and electron/photon identification at a higher granularity provided all of the calorimeter information is available on ROD digitally. Flexible trigger tower information could be formed on ROD digitally before further processed on Level-1 calorimeter trigger system. This will also replace the current bulky copper cable connection between the LAr front-end, the LAr trigger receiver and the Level-1 calorimeter trigger system.

\subsection{Low Voltage Power Supply}

The current front-end power supply system is of centralized architecture with each FEC having its own LVPS which takes $280 \mathrm{~V}$ input from USA15 through $\sim 100 \mathrm{~m}$ cable and provides seven different voltages. A LVPS has high power density $(\sim 3.2 \mathrm{~kW})$ and is water cooled. In addition, each FEB has 19 voltage regulators on board. The new LVPS will have to sustain higher radiation level, even though total power budget of front-end electronics will be kept same. We have to rationalize the number and levels of voltages, reduce different voltages across devices, optimize the design of magnetics, minimize the impact of switching frequency and eliminate the single point of failure. Two possible LVPS archtectures are being investigated.

- Distributed power architecture. A main converter generates distribution bus and on-board point of load (POL) converters directly supply low voltages to loads.

- Intermediate bus architecture. In addition to the generation of main voltage bus, a second set of bus voltages is provided, then lower voltages are given by the POL converters.

\section{Summary}

A new front-end readout architecture has been proposed and is being developed for the ATLAS LAr calorimeter readout electronics upgrade. A good number of R\&D projects of LAr calorimeter readout electronics are being actively worked on, with the aim for the ATLAS phase-2 upgrade around 2017.

\section{References}

[1] G. Aad et al. [ATLAS Collaboration], "The ATLAS Experiment at the CERN Large Hadron Collider," JINST 3 (2008) S08003.

[2] [ATLAS Collaboration], "ATLAS liquid argon calorimeter: Technical design report," CERN-LHCC-96-41

[3] N. J. Buchanan et al., "Design and implementation of the Front End Board for the readout of the ATLAS liquid argon calorimeters," JINST 3, P03004 (2008).

[4] W.E. Cleland and E.G. Stern, "Signal processing considerations for liquid ionization calorimeters in a high rate environment," Nucl. Inst. Meth. A 338 (1994) 467

[5] N. J. Buchanan et al., "ATLAS liquid argon calorimeter front end electronics," JINST 3, P09003 (2008).

[6] A. Bazan et al. [Liquid Argon Back End Electronics Collaboration], "ATLAS Liquid Argon Calorimeter Back End Electronics," JINST 2 (2007) P06002.
[7] N. J. Buchanan et al., "Radiation qualification of the front-end electronics for the readout of the ATLAS liquid argon calorimeters," JINST 3, P10005 (2008) 